\section{Notable advances}

\author{
Some of the key papers published in 2008
}

\section{DIABETES}

Those who hope for a stem cell-based cure for diabetes received a boost from a study in mice. A Boston-based team exposed mice to a modified virus that contained genes encouraging the 'exocrine' cells from the animals' pancreata to become reprogrammed to churn out insulin (Nature 455, 627-632; 2008).

When it comes to the pancreas, all is not lost. Past experiments had indicated that the adult organ no longer has progenitor cells that can give rise to insulin-producing $\beta$-cells, which undergo autoimmune attack in type 1 diabetes. A new mouse study, however, offers evidence that these types of 음 progenitor cells do, in fact, exist in the adult pancreas and can give rise to new $\beta$-cells through laboratory manipulation (Cell 132, 197-207; 2008).
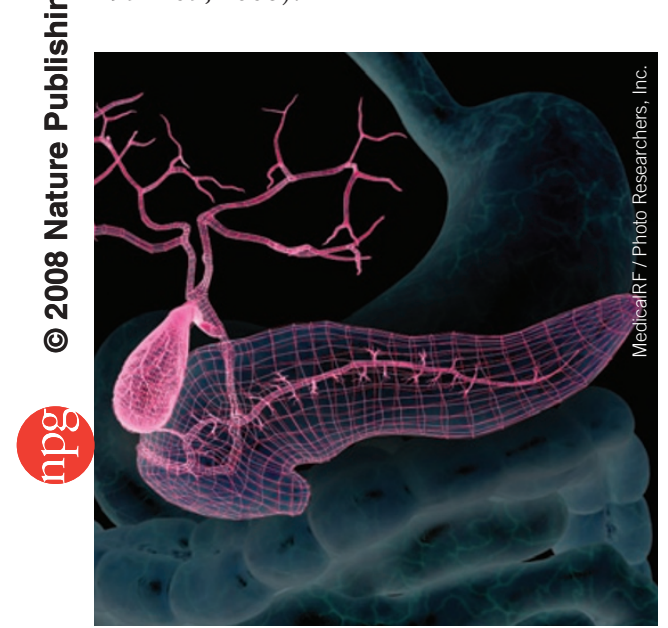

\section{GUT BACTERIA AND DISEASE}

This year, biologists had to stomach the fact that the immune system shares numerous, previously unidentified connections with the gut. For example, one study in mice found that a sugar produced by the common gut bacterium Bacteroides fragilis can prevent intestinal inflammation (Nature 453, 620-625; 2008). Experts say the finding could provide clues about the cause of inflammatory bowel disease, which has increased steadily in the Western world over the past half-century. Another paper reported that the presence of intestinal microbes in a particular strain of mice attenuated the development of type 1 diabetes (Nature 455, 1109-1113; 2008).

\section{- STEM CELLS}

The push to explore ethically acceptable sources of stem cells continued after reports that described how to reprogram human skin cells to mimic embryonic stem cells (Cell 131, 861-872; 2007; Science 318, 1917-1920; 2007). This year, researchers succeeded in turning the clock back on adult cells using minimalist methods: for example, by exposing skin cells to the chemical valproic acid, they bypassed the need to mix in two genes to revert the cells back into a stem cell-like state (Nat. Biotechnol. doi:10.1038/nbt.1502; 2008). Scientists were also able to reprogram adult cells into so-called 'induced pluripotent stem cells' (iPS cells) using genetic elements that, in contrast to the retroviruses previously used for this purpose, do not integrate into the genome, where they can wreak havoc (Science 322, 945-949; 2008; Science 322, 949-953; 2008).

And although the thought of a testicular biopsy may make some men uncomfortable, cells from the human testes seem able to mimic embryonic stem cells when provided with the right growth proteins (Nature, doi:10.1038/ nature 07404; 2008). German scientists ultimately transformed these spermatogonial cells into other cell types, including heart, bone and nerve cells. These cells derived from testicular samples could provide clues about inherited diseases or potentially treat illnesses.

Scientists have for a long time said that one might be able to pinpoint the causes of various ailments by watching patient-derived stem cells develop into different tissue types. They moved one step closer toward this goal in 2008: for the first time, researchers created iPS cells from individuals with disorders such as Parkinson's disease and Down's syndrome (Cell 134, 877886; 2008; Science 321,1218-1221; 2008). The hope is to pinpoint the biological errors that contribute to these diseases by tracking the patient-specific iPS cells' growth in a dish.

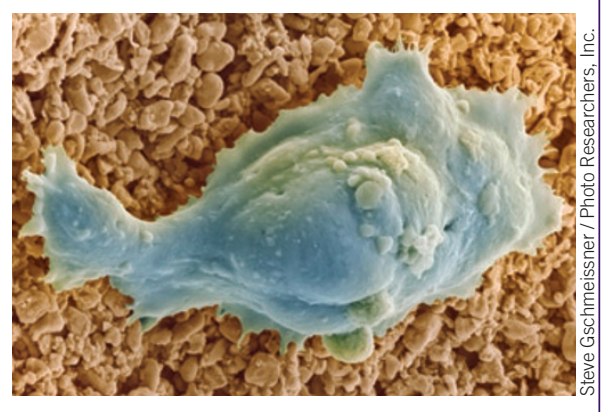

\section{HIV}

Those engaged in the fight against HIV got an unprecedented glimpse into the range of cellular proteins that have a role in virus replication. Scientists used small interfering RNA screens to block the function of individual cellular genes, which revealed the cellular proteins needed for HIV infection and replication. Three studies using this approach identified hundreds of proteins that the virus relies on to replicate efficiently, some of which may prove to be potential new drug targets to treat HIV/ AIDS (Science, doi:10.1126/science.1152725, 2008; Cell 135, 49-60; 2008; Cell Host Microbe doi:10.1016/j.chom.2008.10.004; 2008).

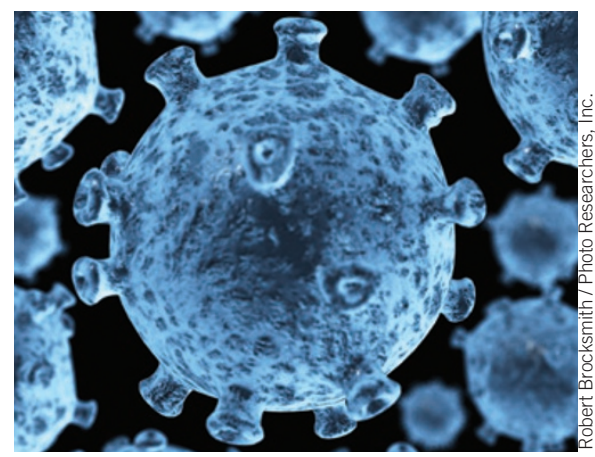

\section{METABOLISM}

When your body breaks down food into sugar, it might set off a feedback loop: scientists discovered a previously unidentified cell signaling pathway in which the presence of circulating glucose boosts glucose production in the liver (Science 319, 1402-1405; 2008). The experiment implicated the enzyme $O$-glycosyl transferase as a culprit in encouraging the liver to make more glucose. The findings could provide clues about why type 2 diabetes worsens over time. Another experiment reported around the same time identified a molecular mechanism by which $O$-glycosyl transferase influences insulin signaling (Nature 451, 964-969; 2008).

When it comes to fat, brown is best. Brown fat helps generate body heat, whereas white fat can weigh you down. Two reports this year shed light on the origins of brown fat. One team found that bone morphogenetic protein-7 (BMP7) acts as a key promoter of brown fat development. The absence of this protein translates into low levels of brown fat in mouse embryos (Nature 454, 1000-1004; 2008). The second study revealed that brown fat shares a common developmental pathway with muscle. Moreover, it identified a molecular switch-PRDM16 - that determines the fate of these cells. An abundance of PRDM16 causes them to develop into brown fat, whereas a lack of it leads them to become muscle (Nature 454, 961-967; 2008). 